\title{
Asymmetric dimethylarginine and asthma
}

\author{
To the Editor:
}

With interest we have read the letter by LAU et al. [1] regarding circulating asymmetric dimethylarginine (ADMA) in a cohort of children with asthma from the Australian Childhood Asthma Prevention Study.

ADMA, a product of protein degradation, is an endogenous nitric oxide synthase inhibitor that has been shown to be increased in mouse models of allergic airway inflammation and to be associated with airways hyperresponsiveness in these animals [2]. ADMA has also been reported to be increased in sputum samples [2] and exhaled breath condensates [3] from paediatric patients with asthma. Increased ADMA in serum has recently been found to be associated with the severity of symptoms in late-onset asthma and obesity [4].

LAU et al. [1] found no difference in serum ADMA between clinically stable children with or without wheezing in the previous 12 months in their cohort of 8-year-old children. However, there was also no difference in post-bronchodilator spirometry (forced vital capacity (FVC) or the per cent predicted (\% pred) forced expiratory volume in $1 \mathrm{~s}\left(\mathrm{FEV}_{1}\right)$ or $\left.\mathrm{FEV} 1 / \mathrm{FVC}\right)$ between the groups, nor was there evidence for increased exhaled nitric oxide fraction ( $\mathrm{FeNO}$ ) in current asthma, as the mean FeNO, although statistically different, was well within the normal range and very similar between the groups (mean \pm SD $8.9 \pm 9.3$ versus 11.1 $\pm 7.7 \mathrm{ppb}$ ). Serum ADMA did not correlate with the (log-transformed) FeNO.

One explanation for the discrepancy between their results and previous reports could be that markers of Larginine/nitric oxide homeostasis in airways may not be altered in the blood of stable patients with mild asthma and normal pulmonary function. This argument is consistent with the findings of Holguin et al. [4], who reported that ADMA levels in peripheral plasma samples from patients with severe, late-onset asthma correlated with worsening of the FEV1 \% pred, FVC \% pred and symptom scores. These data and data from adults with severe asthma [5] suggest that changes in circulating ADMA are associated with specific well-defined phenotypic characteristics that may not manifest with milder disease phenotypes.

We would like to provide additional supporting evidence of the potential role of ADMA in airway responsiveness: we obtained induced-sputum samples from 10 adult subjects with mild, eosinophilic asthma (five males, five females; median age 36 years, range 18-61 years; normal baseline FEV1 of $100.4 \pm 4.7 \%$ pred) at baseline and 7 and 24 hours following a controlled inhaled allergen challenge, as previously described [6, 7]. Baseline sputum eosinophils $(1.6 \pm 0.9 \%)$ increased to $7.6 \pm 3.1 \%$ and $12.5 \pm 6.0 \%$ at 7 and 24 hours following allergen challenge, respectively ( $\mathrm{p}<0.05$ by Friedman test). Neutrophil counts in sputum were $39.0 \pm 6.4 \%$ (maximum $69 \%$ ) at baseline. Changes in pulmonary function testing consistent with airways hyperresponsiveness and airflow limitation (i.e. reduction in the provocative concentration causing a $20 \%$ fall in FEV1 (PC20), and decline in FEV1 \% pred) were observed in these subjects, along with a significant increase in sputum ADMA after challenge $(\mathrm{p}<0.05$ by repeated measures ANOVA) (fig. 1). Thus, in these adult subjects with mild allergic asthma, concentrations of the nitric oxide synthase inhibitor ADMA increase significantly in sputum after allergen challenge, concomitant with the onset of airways obstruction. These data suggest that ADMA is involved in the asthmatic response and that changes in
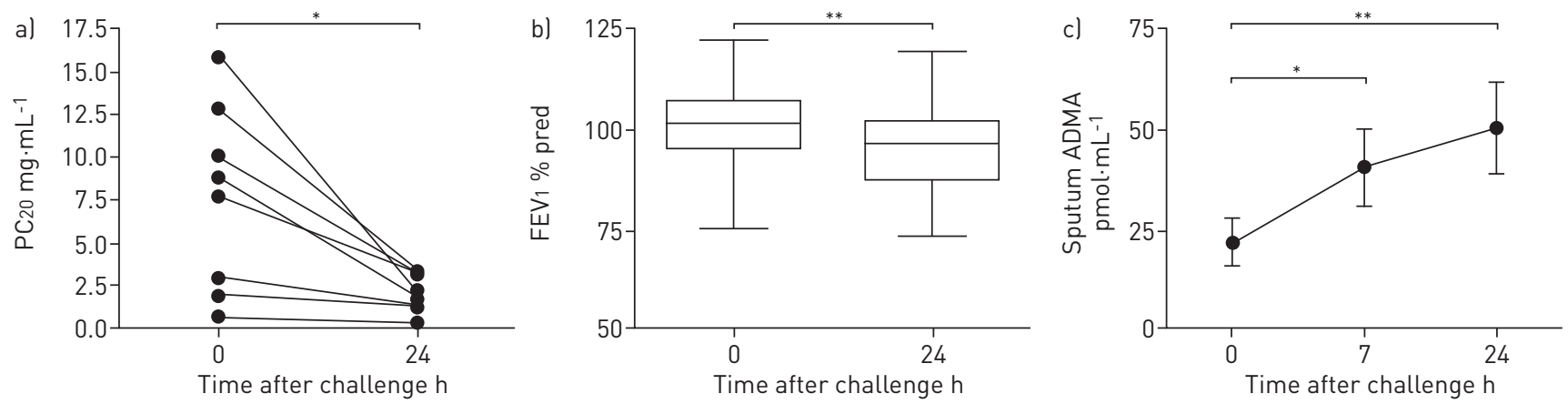

FIGURE 1 a) Methacholine provocative concentration causing a $20 \%$ fall in forced expiratory volume in $1 \mathrm{~s}$ (PC20) is reduced in asthmatic subjects $24 \mathrm{~h}$ after specific allergen challenge. b) Forced expiratory volume in $1 \mathrm{~s}$ (FEV1) \% predicted is similarly attenuated at $24 \mathrm{~h}$ after allergen challenge, compared with the baseline (pre-challenge). c) A significant increase in sputum asymmetric dimethylarginine (ADMA) concentration is observed at 7 and $24 \mathrm{~h}$ after allergen challenge. $\mathrm{n}=8$ in each group. ${ }^{\star}: \mathrm{p}<0.05 ;{ }^{* *}: \mathrm{p}<0.01$. 
ADMA in the airways occur in acute exacerbations. It will be very interesting to see what future studies comparing the utility of biomarkers between different compartments will tell us about the role of ADMA in asthma.

0 @ERSpublications

Sputum ADMA increases in mild allergic asthmatics after allergen challenge, concomitant with onset of airways obstruction http://ow.ly/nM3VV

Jeremy A. Scott ${ }^{1,2}$, Gail M. Gauvreau ${ }^{3}$ and Hartmut Grasemann ${ }^{4}$

${ }^{1}$ Dept of Health Sciences, Lakehead University, Thunder Bay, ON, ${ }^{2}$ Division of Medical Science, Northern Ontario School of Medicine, Thunder Bay and Sudbury, ON, ${ }^{3}$ Division of Respirology, Dept of Medicine, McMaster University, Hamilton, ON, and ${ }^{4}$ Program in Physiology and Experimental Medicine, Research Institute, and Division of Respiratory Medicine, Dept of Paediatrics, University of Toronto, Toronto, ON, Canada.

Correspondence: J.A. Scott, Dept of Health Sciences, Lakehead University, 955 Oliver Road, Thunder Bay, ON, P7B 5E1, Canada. E-mail: jascott1@lakeheadu.ca

Received: May 082013 | Accepted after revision: May 172013

Conflict of interest: None declared.

\section{References}

Lau EMT, Morgan PE, Belousova EG, et al. Asymmetric dimethylarginine and asthma: results from the Childhood Asthma Prevention Study. Eur Respir J 2013; 41: 1234-1237.

2 Scott JA, North ML, Rafii M, et al. Asymmetric dimethylarginine is increased in asthma. Am J Respir Crit Care Med 2011; 184: 779-785.

3 Carraro S, Giordano G, Piacentini G, et al. Asymmetric dimethylarginine in exhaled breath condensate and serum of children with asthma. Chest 2013; 144: 405-410.

4 Holguin F, Comhair SA, Hazen SL, et al. An association between L-arginine/asymmetric dimethyl arginine balance, obesity, and the age of asthma onset phenotype. Am J Respir Crit Care Med 2013; 187: 153-159.

5 Lara A, Khatri SB, Wang Z, et al. Alterations of the arginine metabolome in asthma. Am J Respir Crit Care Med 2008; 178: 673-681.

6 Boulet LP, Gauvreau G, Boulay ME, et al. The allergen bronchoprovocation model: an important tool for the investigation of new asthma anti-inflammatory therapies. Allergy 2007; 62: 1101-1110.

7 Pizzichini E, Pizzichini MM, Efthimiadis A, et al. Indices of airway inflammation in induced sputum: reproducibility and validity of cell and fluid-phase measurements. Am J Respir Crit Care Med 1996; 154: 308-317.

\section{Early-life wheeze: "the Child is father of the Man"}

To the Editor:

We applaud the recent article by HovLAND et al. [1] from the Environment and Childhood Asthma (ECA) study, which provides intriguing insight into the longer-term consequences of early-life wheezing. Employing a different classification of early wheeze (recurrent bronchial obstruction (RBO)) to previous studies, including our own Isle of Wight Birth Cohort (IOWBC), their findings suggest that a notinsubstantial proportion of such disease is associated with wheezing at the end of childhood. We note their comparison of findings with those reported from the 10-year follow-up of our IOWBC, highlighting both similarities and differences between the two studies. They also query what a later follow-up of the IOWBC would show in relation to young adult outcome of early-life wheezing. The ECA report followed up patients at 16 years of age, whereas the corresponding follow-up of our cohort was at 18 years. Using data from that follow-up, we can both corroborate the findings of HovLand et al. [1] and shed further light on their understanding by returning to our earlier classification of wheeze phenotypes in the first decade of life.

Hovland et al. [1] used a very stringent definition of "early life" confined to wheezing events in the first 2 years of life. By contrast, the IOWBC papers they compare their findings against use a broader definition of "early life" encompassing the first 4 years of life [2,3]. Interest is growing in the influence of early life as a period of vulnerability for disease development and an argument could be made that the approach of Hovland et al. [1] better captures that period. With a wider timespan, we previously found prevalence for early life (in the first 4 years) wheezing of $40 \%$ at 10 years in our cohort [2]. We also showed that $37 \%$ of 